\title{
Cost-effectiveness analysis of different types of human papillomavirus vaccination combined with a cervical cancer screening program in mainland China
}

Xiuting Mo ${ }^{1,2}$, Ruoyan Gai Tobe ${ }^{2 *}$, Lijie Wang ${ }^{3}$, Xianchen Liư 4 , Bin Wu ${ }^{5}$, Huiwen Luo ${ }^{6}$, Chie Nagata ${ }^{7}$, Rintaro Mori ${ }^{2}$ and Takeo Nakayama ${ }^{1}$

\begin{abstract}
Background: China has a high prevalence of human papillomavirus (HPV) and a consequently high burden of disease with respect to cervical cancer. The HPV vaccine has proved to be effective in preventing cervical cancer and is now a part of routine immunization programs worldwide. It has also proved to be cost effective. This study aimed to assess the cost-effectiveness of 2-, 4-, and 9-valent HPV vaccines (hereafter, HPV2, 4 or 9) combined with current screening strategies in China.
\end{abstract}

Methods: A Markov model was developed for a cohort of 100,000 HPV-free girls to simulate the natural history to HPV infection. Three recommended screening methods (1. liquid-based cytology test + HPV DNA test; 2 . pap smear cytology test + HPV DNA test; 3 . visual inspection with acetic acid) and three types of HPV vaccination program (HPV2/4/9) were incorporated into 15 intervention options, and the incremental cost-effectiveness ratio (ICER) was calculated to determine the dominant strategies. Costs, transition probabilities and utilities were obtained from a review of the literature and national databases. One-way sensitivity analyses and threshold analyses were performed for key variables in different vaccination scenarios.

Results: HPV9 combined with screening showed the highest health impact in terms of reducing HPV-related diseases and increasing the number of quality-adjusted life years (QALYS). Under the current thresholds of willingness to pay (WTP, 3 times the per capita GDP or USD\$23,880), HPV4/9 proved highly cost effective, while HPV2 combined with screening cost more and was less cost effective. Only when screening coverage increased to $60 \% \sim 70 \%$ did the HPV2 and screening combination strategy become economically feasible.

Conclusions: The combination of the HPV4/9 vaccine with current screening strategies for adolescent girls was highly cost-effective and had a significant impact on reducing the HPV infection-related disease burden in Mainland China.

Keywords: Cervical cancer, Cervical intraepithelial neoplasia, Incremental cost-effectiveness ratio, Vaccine

\footnotetext{
* Correspondence: gai-r@ncchd.go.jp

${ }^{2}$ Department of Health Policy, National Center for Child Health and

Development, Okura 2-10-1, Setagaya-ku, Tokyo 157-8535, Japan

Full list of author information is available at the end of the article
} 


\section{Background}

Cervical cancer is one of the most widespread gynaecological cancers worldwide and remains the second leading cause of gynaecological-related mortality. More than $85 \%$ of cases occur in developing countries, resulting in an estimated 275,000 deaths annually [1]. In mainland China, despite its decreasing incidence in recent years, cervical cancer remains among the top ten most common malignancies among women and is a leading cause of cancer-related mortality nationwide [2, 3]. Human papillomavirus (HPV) infection is the main cause of cervical cancer and can be detected in more than 95\% of uterine carcinomas. Vaccinations are effective in preventing HPV infection, and a number of vaccine options have recently become available. The 2 -valent $\mathrm{HPV}$ vaccine (HPV2), which targets HPV 16/18 and the 4-valent HPV vaccine (HPV4), which targets 6/11/16/18, have been approved in more than 100 countries worldwide. Both vaccines were highly effective in clinical trials and shown to be cost effective in health economic studies in various countries [4]. The 9-valent HPV vaccine (HPV9) targeting five additional oncogenic HPV types (31, 33, 45, 52, and 58) improved protection against $90 \%$ of cervical cancers, an increase of $20 \%$ from the previous figure, and has been licensed in more than 30 countries [5, 6]. In July 2016, a decade after international approval of the first HPV vaccine, the Chinese government approved GSK Cervarix ${ }^{\mathrm{TM}}$, a 2-valent HPV vaccine expected to become commercially available in early 2017 [7, 8]. In addition, some other HPV-related cancers (such as oropharyngeal, anal, and vulvar cancer) caused by HPV 16/18/31/33/45/ $52 / 58$ may be prevented by the vaccines [9-11].

Further, as recommended by the World Health Organization (WHO), screening is a common preventative strategy targeting women of reproductive age for the early detection and treatment of HPV infection, cervical cancer, and cervical intraepithelial neoplasia (CIN). In developing countries, routine screening programs reportedly reduced the incidence of cervical cancer by up to $60 \%$ [12]. In 2005 the Ministry of Health of China formed guidelines for the screening, early detection, and treatment of cervical cancer [13]. The target demographic of these guidelines was women older than 21 years and those who engaged in sexual activity for more than three years. The guidelines recommend three different protocols each designed for a particular socioeconomic stratum: 1) primary screening by liquid-based cytology test + HPV DNA test, which has optimal sensitivity and specificity but is expensive and requires wellequipped infrastructure and ample resources; 2) primary screening by pap smear cytology test + HPV DNA test, requiring less infrastructure and resources; and 3) primary screening by visual inspection with acetic acid (VIA), a basic protocol designed for low income settings.
Although a government-sponsored VIA and cytology screening program has been carried out in some regions [14], no national cervical screening program currently exists on mainland China. According to some local surveys, the coverage rate of current screening programs is approximately $10 \sim 30 \%[15,16]$ and even when offered free of charge, remained at around 50\% [17].

Although the cost-effectiveness of the HPV vaccine in China has been analysed [18-20], a thorough assessment specific to China of HPV2, 4, and 9 and the three previously mentioned screening protocols still remains to be done. Therefore, this study aims to evaluate the costeffectiveness of different HPV vaccinations as alternatives, and as an adjunct to the three primary screening strategies currently in use in mainland China, with consideration of diversified geographical characteristics.

\section{Methods}

\section{Overview of the model}

A Markov model was used to simulate the natural history of HPV infection and to estimate the economic consequences of HPV-related diseases from a societal perspective view (Fig. 1). The rationale for using the model was the example provided by several previous natural history models of HPV simulating high and low risk HPV infections separately. In this study, the model was adapted to the Chinese context (three different screening strategies and diagnosis/ treatment flow specific to the Chinese setting) in order to reflect local screening and treatment practices [21-23].

We assumed that girls under 12 years old were virgins. In our analysis, we simulated a cohort of 100,000 HPVfree girls (from 12 years old and followed up until death). In the strategies using vaccination, individuals were given the respective vaccines at the beginning of the simulation. HPV genotypes were divided into high risk (carcinogenic HPV 16/18/31/33/45/52/58, etc.) and low risk (HPV 6/11 and etc.) groups in the analysis. Health states were defined based on the natural history of HPV infection in the following manner: healthy, low risk HPV infection, high risk HPV infection, mild cervical intraepithelial neoplasia (CIN 1) caused by high risk HPV infection, moderate cervical intraepithelial neoplasia (CIN 2) caused by high risk HPV infection, severe cervical intraepithelial neoplasia or carcinoma in situ (CIN 3) caused by high risk HPV infection, CIN1/2 caused by low risk HPV infection, genital warts, invasive cervical cancer (ICC) (stages I-IV as defined by the International Federation of Gynaecology and Obstetrics, FIGO), cervical cancer survival, death from cervical cancer, and death from other causes [21]. Oropharyngeal, anal, and vulvar cancers were not included in this study because differing proportions of these cancers are caused by HPV, and it is difficult to quantify the effects of vaccination on the incidence of HPV caused by the strains 


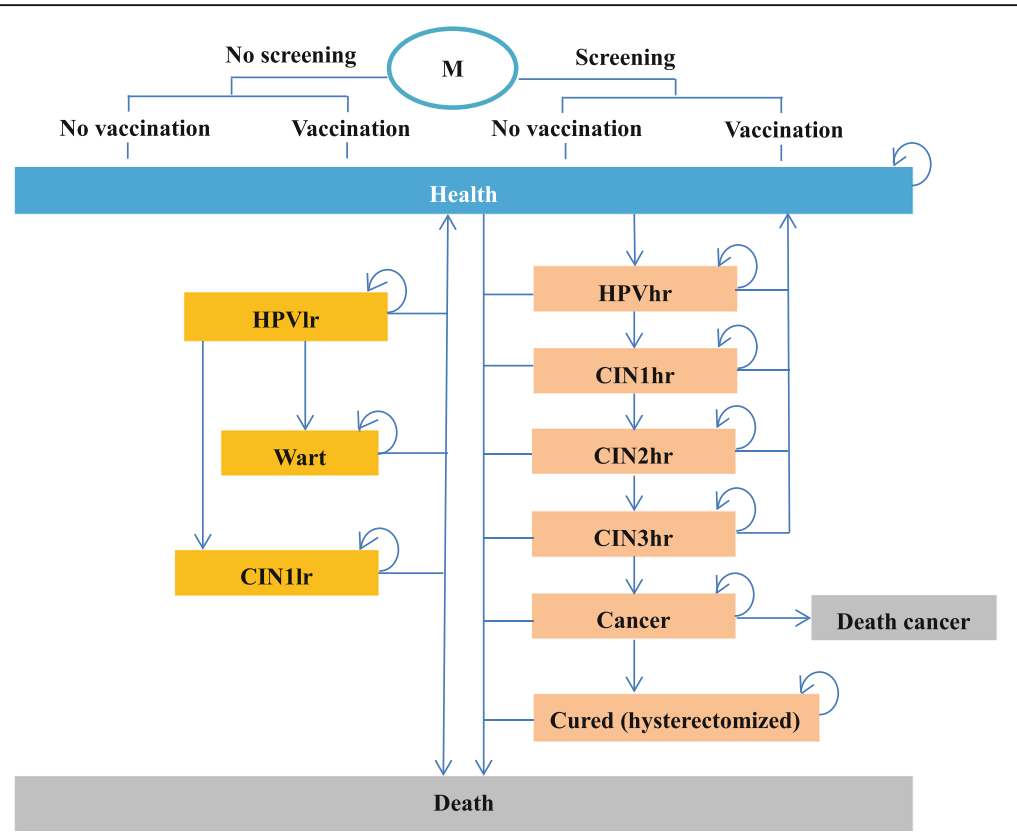

Fig. 1 Markov model of the history of high and low risk type of HPV. The arrows direct transitions from one state to another. hr, high-risk; Ir, low-risk; CIN, cervical intraepithelial neoplasia; HPV, human papillomavirus

covered. Furthermore, the epidemiological data on HPVrelated cancers other than cervical cancer caused by HPV $16 / 18$ or HPV 16/18/31/33/45/52/58 were not available in China. Since the impact of the HPV vaccines on other non-cervical HPV-related cancers have not been included in the analysis, the projected health benefits are likely underestimated.

The Markov model developed for this study comprehensively assessed relevant and currently in-use preventative strategies including both vaccination and screening in China. The screening strategies included the three protocols recommended in the 2005 Guidelines for Screening, Early Detection and Treatment of Cervical Cancer [13]: 1) liquid-based cytology test + HPV DNA test; 2) pap smear cytology test + HPV DNA test; 3) visual inspection with acetic acid (VIA) and a colposcopy following a positive result to make a definite diagnosis. The vaccination options were HPV2 (CERVARIX,HPV4 (GARDASIL ${ }^{\circ}$ ) and the newly developed HPV9 (GARDASIL ${ }^{\circ}$ ). We compared 15 preventative strategies consisting of either a single modality or a combination of vaccination and screening, namely, Screening 1, Screening 2, Screening 3, HPV2, HPV4, HPV9, Screening $1+$ HPV2, Screening $2+$ HPV2, Screening $3+$ HPV2, Screening $1+$ HPV4, Screening $2+$ HPV4, Screening $3+$ HPV4, Screening $1+$ HPV9, Screening $2+$ HPV9, Screening $3+$ HPV9, and no intervention.

\section{Screening strategies}

The target population for screening was women older than 21 years. Screening every three years was recommended.
Based on the cervical cancer prevention guidelines of mainland China and several Chinese reports, we assumed that a liquid-based cytology test would have a sensitivity of 0.85 and a specificity of 0.90 [24]. The HPV DNA test had a sensitivity of 0.95 and specificity of 0.85 [24]. The pap smear had a sensitivity of 0.65 and specificity of 0.88 [24]. The VIA test had a sensitivity of 0.68 and specificity of 0.85 [24-26]. We assumed that the colposcopy + biopsy combination would have perfect sensitivity and specificity for the detection of CIN2+ [27].

A screening coverage rate of $20 \%$ was assumed due to the large geographical diversity in socioeconomic development, and $10 \%-100 \%$ was set for the sensitivity analysis [28].

\section{Vaccine characteristics}

As vaccine efficacy data specific to the Chinese population were unavailable, the data used here were obtained by multiplying the direct efficacy targeting specific HPV types by serotype coverage on mainland China. In the clinical trials, HPV2 (CERVARIX),HPV4 (GARDASIL ${ }^{\circ}$ ), and HPV9 (GARDASIL ${ }^{\circ}$ ) all showed excellent efficacy against the relevant CIN and AIS (Adenocarcinoma in situ) in women with HPV DNA negative for oncogenic HPV types at baseline: HPV2: 96.5\% (95\% CI: 91.6\%98.9\%) [29]; HPV4: 96.0\% (95\% CI: 92.3\%-98.2\%) [10]; HPV9: 96.0\% (95\% CI: 92.3\%-98.2\%) for HPV 6, 11, 16, or 18-related CIN or AIS, and 96.7\% (95\% CI: 80.9\%99.8\%) for the additional five HPV types, namely, HPV $31,33,45,52$, and 58 [11]. The efficacy of the HPV4 and HPV9 against specific HPV-related genital warts was 
99.0\% (95\% CI: 96.2\%-99.9\%) [11]. HPV2 was assumed to have the same efficacy against genital warts $(99.0 \%$, 95\% CI: 96.2\%-99.9\%). The parameter: HPV serotype coverage of each vaccine, was obtained from a doctoral dissertation, an epidemic research on HPV distribution in genital warts, ICC and CIN2/3 samples from 18 hospitals in 7 geographic regions [30]. HPV6, 11 and 16 distribution in genital warts patients were not significantly differed by urban and rural areas. Nor did HPV prevalence in squamous cell carcinoma patients differ notably by region [30]. The following are the HPV types with their respective incidence for CIN2/3: HPV 6: 1.26\%; HPV 16: 75.94\%; HPV 11: 0; HPV 18: 7.70\%; HPV 31: 3.14\%; HPV 33: 0.94\%; HPV 45: 1.10\%; HPV 52: 2.20\%; and HPV 58: $2.20 \%$ [30]. The rates for genital warts were HPV 6: 46.6\%; HPV 11: 42.4\%, and HPV 16: $11.8 \%$ [30]. The parameters for the sensitivity analysis were the baseline value $\pm 25 \%$.

After calculation, the overall efficacy of HPV2, HPV4, and HPV9 against cervical cancer was $80.72 \%(57.47 \%-$ 98.9\%), 81.51\% (58.78\%-98.2\%), and 90.78\% (66.54\%$100 \%)$, respectively, and that against genital warts was $10.36 \%$ (7.56\%-13.07\%), 86.06\% (62.72\%-99.99\%) and $86.06 \%$ (62.72\%-99.99\%), respectively.

Moreover, considering the reported efficacy data and demonstration of immunological memory [31], we assumed that the protection conferred by the vaccines would be life-long and obviate the need for a booster. Because of the relatively low coverage of the current prevention program, cross protective effects were not taken into account.

A vaccine coverage rate was assumed as $20 \%$, and $10 \%-100 \%$ was set for the sensitivity analysis.

\section{Transition probabilities}

Each strategy (screening plus vaccination or screening only) modelled probabilities for mutually exclusive health states within a one-year cycle. At each transition, the model produced figures for the costs incurred and the QALYs according to the individual's health condition. Each year, the target population was exposed to an agespecific risk of HPV infection that could persist, progress (to CIN 1, CIN 2-3, genital warts, etc.) or resolve. In those who developed CIN 3, their disease might persist, remit, or progress to localized (FIGO stage I and IIA), regional (FIGO IIB to IVA) or metastatic invasive cancer (FIGO IVB). Given the course of cervical cancer, each individual might continue to suffer from the disease, die, or experience complete remission. Each year individuals faced agespecific risks of dying from other causes or of undergoing a hysterectomy for reasons unrelated to cervical neoplasia. Women who underwent a hysterectomy did not develop cervical cancer in our model. Table 1 summarizes the initial value and all the transition probabilities derived from the previous reports and research projects of the Cancer Institute and Hospital, Chinese Academy of Medical Sciences (CICAMS). Screening Technologies to Advance Rapid Testing for Cervical Cancer Prevention (START) was calibrated to reflect Chinese epidemiology [32].

\section{Health utilities}

Utilities are a measure of the quality of life rated on a scale of 0 (death) to 1 (optimal health), and are based on economic studies in China. CIN 2 was assigned a lower utility (0.88) [33], while CIN 3 was assigned an utility of 0.81 for a 1-year period [33]. In cases of ICC, a woman's utility was assumed to decrease to 0.69 [33]. The utility of cervical cancer survivors was 0.85 [28, 34, 35]. The utility of patients with genital warts was determined to be 0.67 based on some Chinese studies [36, 37].

\section{Cost estimates}

Cost estimates included the cost of vaccination, screening, and treatment of diseases related to HPV infection. Data were initially calculated in Chinese Yuan and then converted to US dollars in 2015 using the consumer price index (CPI) and the official exchange rate of the Chinese Yuan to US dollar (January 2015 exchange rate, USD\$1 = CYN6.20). Although the three HPV vaccines mentioned above were not yet available on mainland China, Gardasil, a quadrivalent vaccine developed by Merck and Cervarix, a bivalent vaccine developed by GlaxoSmithKline, both costing about the same, were approved in Hong Kong. We estimated the costs of three doses according to the standard price in Hong Kong $(2000 \sim 3000 \mathrm{CYN})$ [38], and the administration fee for each girl was estimated at USD $\$ 4.84$ dollars. Cost of three doses of HPV9 vaccine was calculated by multiplying USD\$ 403.23 by the ratio of cost of HPV4/HPV9 per dose $(\$ 147.78 / \$ 163.86=1.1088)$ [39]. Considering the likelihood that the costs on the mainland would be lower than in Hong Kong due to the lower economic status of the mainland population, the sensitivity analysis was performed with a value range of $0.5 \mathrm{X}-1.5 \mathrm{X}$. Moreover, we approximated different screening costs using the current national tariff proposed by the Chinese AntiCancer Association [40]. The treatment costs were obtained from tertiary hospitals and the local health system. Treatment by FIGO staging was based on the recommendation of national and international guidelines [41]. A half cycle correction was used in order to achieve a closer approximation to proper reward/survival.

In terms of compliance, the baseline value for CIN $2 / 3$ treatment extracted from Screening Technologies to Advance Rapid Testing for Cervical Cancer Prevention (START) was set at 90\% [28]. The selection rate for a hysterectomy in the event of CIN 3 was set at $20 \%$ for those older than 35 years and $0 \%$ for all others [28]. When 
Table 1 Model variables: Baseline values and ranges used in sensitivity analysis

\begin{tabular}{|c|c|c|c|}
\hline Variable & Base case & Plausible range & References \\
\hline Natural history of HPV & Age-specific table & & \\
\hline HPV-2 & Serotypes covered*efficacy & & \\
\hline Efficacy for cervical cancer & $0.836^{*} 0.965$ & $0.575-0.989$ & {$[27,30]$} \\
\hline Efficacy for genital warts & $0.107^{*} 0.990$ & $0.756-0.131$ & Assumed \\
\hline \multicolumn{4}{|l|}{ HPV-4 } \\
\hline Efficacy for cervical cancer & $0.849^{*} 0.960$ & $0.588-0.982$ & {$[28,30]$} \\
\hline Efficacy for genital warts & $0.869^{*} 0.990$ & $0.627-0.999$ & {$[29,30]$} \\
\hline \multicolumn{4}{|l|}{ HPV-9 } \\
\hline Efficacy for cervical cancer & $0.849^{*} 0.960+0.096^{*} 0.967$ & $0.665-1.000$ & {$[29,30]$} \\
\hline Efficacy for genital warts & $0.869^{*} 0.990$ & $0.627-0.999$ & {$[29,30]$} \\
\hline \multicolumn{4}{|l|}{ Pap smear } \\
\hline Sensitivity & 0.65 & $0.50-0.80$ & [22] \\
\hline Specificity & 0.88 & $0.85-0.90$ & {$[22]$} \\
\hline \multicolumn{4}{|l|}{ Liquid-based cytology test } \\
\hline Sensitivity & 0.85 & $0.80-0.90$ & {$[22]$} \\
\hline Specificity & 0.90 & $0.85-0.95$ & [22] \\
\hline \multicolumn{4}{|l|}{ HPV DNA test } \\
\hline Sensitivity & 0.95 & $0.80-0.98$ & [22] \\
\hline Specificity & 0.85 & $0.80-0.90$ & [22] \\
\hline \multicolumn{4}{|l|}{ VIA } \\
\hline Sensitivity & 0.68 & $0.50-0.70$ & {$[22-24]$} \\
\hline Specificity & 0.85 & $0.66-0.96$ & {$[22-24]$} \\
\hline \multicolumn{4}{|l|}{ Colposcopy and biopsy } \\
\hline Sensitivity & 1 & $0.50-1.0$ & {$[25]$} \\
\hline Specificity & 1 & $0.50-1.0$ & {$[25]$} \\
\hline Age begin to screen & 20 & $18-45$ & Assumed \\
\hline Screening intervals & 3 & $1,3,5,10$ & Assumed \\
\hline Screening coverage & 0.2 & $0.1-1.0$ & Assumed \\
\hline Vaccine coverage & 0.2 & $0.1-1.0$ & Assumed \\
\hline \multicolumn{4}{|l|}{ Costs (USD) } \\
\hline HPV 2/4 vaccine (3 does) & 403.23 & $0.5 X-1.5 X$ & [38] \\
\hline HPV 9 vaccine (3 does) & 447.10 & $0.5 X-1.5 X$ & {$[38,39]$} \\
\hline vaccine administration (3 does) & 4.84 & $0.5 X-1.5 X$ & Chinese Anti-Cancer Association \\
\hline Pap smear & 6.75 & $0.5 X-1.5 X$ & Chinese Anti-Cancer Association \\
\hline Liquid-based cytology & 43.89 & $0.5 X-1.5 X$ & Chinese Anti-Cancer Association \\
\hline HPV DNA test & 56.45 & $0.5 X-1.5 X$ & Chinese Anti-Cancer Association \\
\hline VIA & 5.06 & $0.5 X-1.5 X$ & Chinese Anti-Cancer Association \\
\hline Colposcopy and biopsy & 32.26 & $0.5 X-1.5 X$ & Local field study \\
\hline Loop electrosurgical excision procedure (LEEP) & 403.23 & $0.5 X-1.5 X$ & Local field study \\
\hline Cold knife conisation & 887.10 & $0.5 X-1.5 X$ & Local field study \\
\hline Hysterectomy & 2419.35 & $0.5 X-1.5 X$ & Local field study \\
\hline Localized cancer & 3225.81 & $0.5 X-1.5 X$ & Local field study \\
\hline Regional cancer & 4838.71 & $0.5 X-1.5 X$ & Local field study \\
\hline Metastatic cancer & 6451.61 & $0.5 X-1.5 X$ & Local field study \\
\hline
\end{tabular}


Table 1 Model variables: Baseline values and ranges used in sensitivity analysis (Continued)

\begin{tabular}{llll}
\hline Genital warts & 161.29 & $0.5 X-1.5 X$ & Local field study \\
Utilities & & & {$[33]$} \\
CIN1 & 0.9965 & $0.992603-1.0$ & {$[33]$} \\
CIN2 & 0.984 & $0.876-1.0$ & {$[33]$} \\
CIN3 & 0.984 & $0.806-1.0$ & {$[33]$} \\
Cancer & 0.693 & $0.56-0.76$ & {$[36]$} \\
Genital warts & 0.827 & $0.701-0.933$ & {$[26,34,35]$} \\
Cancer survival & 0.850 & $0.82-0.88$ & {$[26]$} \\
Compliance of treatment & & & Assumed \\
CIN2+ & 0.9 & & {$[26]$} \\
Cancer & 1.0 & & Assumed \\
Choose hysterectomy when CIN3 & 0.2 when $>35$ & $0-6 \%$ & Assumed \\
Discount rate of cost & $3 \%$ & $0-6 \%$ & \\
Discount rate of effectiveness & $3 \%$ & & \\
\hline
\end{tabular}

cervical cancer was detected or symptoms appeared, compliance with treatment was assumed to be $100 \%$.

\section{Outcome measures}

The model was programmed using TreeAge Pro 2011. We expressed the results in terms of the number of cases of cervical cancer, genital warts, and deaths from cervical cancer prevented, as well as the lifetime cost and QALYs gained. The incremental cost-effectiveness ratio (ICER) was calculated as incremental cost divided by the QALYs gained per woman by adding vaccination to the status quo (i.e., each of the three different screening strategies reflecting three different Chinese settings). According to the WHO, if the ICER is less than the per capita gross domestic product (GDP) a strategy can be considered as "very cost effective", and if less than three times the per capita GDP, as "cost effective" [42]. Three times the 2015 per-capita GDP for China amounted to USD $\$ 23,879.52$ [43]. An annual discount rate of $3 \%$ was applied to both costs and benefits.

\section{Sensitivity analyses}

One-way sensitivity analysis was performed to explore the impact of the uncertainty of the parameters, taking into account simultaneous changes within the plausible ranges. The efficacy of each vaccine, the sensitivity and specificity of different tests, and the utilities associated with different health stages varied uniformly within the referred ranges. The cost parameters obtained varied uniformly in a range of $+/-50 \%$ of the deterministic estimate. The discount rate for cost and effectiveness varied uniformly between 0 to $6 \%$. According to the results of tornado diagrams, threshold analyses were performed.

\section{Results}

\section{Predicted cervical cancer mortality}

Cervical cancer mortality in the trial (screening only without vaccination) was based on cancer statistics. The predicted cervical cancer mortality showed acceptable correspondence with data from the 2011 Chinese Cancer Registry Annual Report [44] (Additional file 1: Figure S1).

\section{Base case analysis}

The cost-effectiveness analysis and the reduction in cervical cancer and warts for each strategy under base case assumptions based on the Monte Carlo simulation of 100,000 trials showed that among the 15 options consisting either of a single strategy or one combining vaccination and screening, HPV9 combined with screening yielded the highest number of QALYs. Screening 1 and Screening 2 and their respective combination strategies showed little difference in discounted QALYs but an obvious cost difference (Table 2). In the calculation of ICERs, we first compared each single strategy (vaccine or screening) with no intervention strategy. Among three different vaccines, HPV4 cost least and gained almost the same number of QALYs as HPV9, while HPV-2 was dominated by HPV-4 due to higher costs and lower effectiveness. Screening 3, on the other hand, cost least, gained nearly the same QALYs as the other two screenings, and showed the most cost-effectiveness, while Screen 1 showed no cost-effectiveness due to its ICER is over 3 times WTP. Then we compared each combination strategy in three different screening settings. HPV2 in its respective combination strategies were always ruled out by absolute dominance (higher cost and lower effectiveness) and demonstrated no costeffectiveness while the HPV4 and HPV9 combination strategies presented significant cost-effectiveness (each 
Table 2 Cost-effectiveness analysis and reduction in cervical cancer and warts of each strategy under base-case assumptions based on Monte Carlo simulation of 100,000 trails

\begin{tabular}{|c|c|c|c|c|c|c|c|}
\hline Strategy & $\begin{array}{l}\text { Discounted } \\
\text { costs }\end{array}$ & $\begin{array}{l}\text { Discounted } \\
\text { QALYs }\end{array}$ & $\begin{array}{l}\text { IC/IE } \\
\text { (\$/QALYS) }\end{array}$ & $\begin{array}{l}\text { Cancer reduction } \\
\text { incidence (\%) }\end{array}$ & $\begin{array}{l}\text { Cancer mortality } \\
\text { reduction (\%) }\end{array}$ & $\begin{array}{l}\text { HPVhr incidence } \\
\text { reduction (\%) }\end{array}$ & $\begin{array}{l}\text { Warts incidence } \\
\text { reduction (\%) }\end{array}$ \\
\hline No intervention & 25.359 & 30.858 & - & 1893.000 & 348.000 & $895,739.000$ & $100,676.000$ \\
\hline$H P V-4^{\mathrm{a}}$ & 101.631 & 30.872 & $5400.550^{f}$ & 15.850 & 18.200 & 18.130 & 15.570 \\
\hline$H P V-2^{a}$ & 103.205 & 30.859 & Dominated & 13.100 & 13.100 & 4.170 & 14.500 \\
\hline$H P V-9^{a}$ & 110.203 & 30.873 & $5768.350^{f}$ & 16.270 & 18.410 & 20.450 & 15.560 \\
\hline Screen $1^{b, c}$ & 116.544 & 30.867 & $\begin{array}{l}959,735.859^{h} \\
9287.599^{9}\end{array}$ & 18.860 & 19.020 & 10.400 & 5.900 \\
\hline Screen $1+\mathrm{HPV}-4^{\mathrm{C}}$ & 193.106 & 30.880 & $6070.026^{f}$ & 33.860 & 35.690 & 23.640 & 20.780 \\
\hline Screen $1+$ HPV- $2^{c}$ & 194.301 & 30.869 & Dominated & 32.280 & 32.280 & 6.490 & 19.840 \\
\hline Screen $1+$ HPV-9 ${ }^{c}$ & 201.724 & 30.881 & $6275.190^{f}$ & 34.390 & 35.950 & 25.820 & 20.800 \\
\hline Screen $2^{b, d}$ & 82.945 & 30.867 & $\begin{array}{l}20,372.19^{9} \\
5886.421^{f}\end{array}$ & 18.860 & 19.020 & 10.340 & 5.880 \\
\hline Screen2 + HPV-4 ${ }^{d}$ & 159.397 & 30.880 & $6058.622^{f}$ & 33.860 & 35.690 & 23.610 & 20.730 \\
\hline Screen2 + HPV-2 ${ }^{d}$ & 160.713 & 30.869 & Dominated & 32.280 & 32.280 & 6.460 & 19.790 \\
\hline Screen2 + HPV-9 ${ }^{d}$ & 168.002 & 30.881 & $6262.947^{f}$ & 34.390 & 35.950 & 25.800 & 20.750 \\
\hline Screen $3^{b, e}$ & 34.004 & 30.865 & $\begin{array}{l}1171.435^{f} \\
1171.435^{f}\end{array}$ & 18.540 & 18.860 & 8.010 & 4.100 \\
\hline Screen3 + HPV-4 & 110.341 & 30.878 & $5865.854^{f}$ & 32.750 & 35.130 & 21.650 & 19.220 \\
\hline Screen3 + HPV-2 $2^{\mathrm{e}}$ & 111.804 & 30.866 & Dominated & 31.170 & 31.720 & 3.890 & 18.280 \\
\hline Screen3 + HPV-9e & 118.927 & 30.879 & $6098.722^{f}$ & 33.280 & 35.400 & 23.910 & 19.230 \\
\hline
\end{tabular}

ICERs were calculated in 5 settings: ${ }^{a}$ no intervention, HPV-4, HPV-2 and HPV-9; ${ }^{b}$ no intervention, Screen3, Screen2 and Screen1; ${ }^{c}$ no intervention, Screen1, Screen 1 + HPV-4, Screen1 + HPV-2, and Screen1 + HPV-9; ${ }^{d}$ o intervention, Screen2, Screen2 + HPV-4, Screen2 + HPV-2, and Screen2 + HPV-9; ${ }^{e}$ no intervention, Screen3, Screen3 + HPV-4, Screen3 + HPV-2, and Screen3 + HPV-9

${ }^{f}$ If $0<$ ICER < per capita GDP (7960 USD), it is considered very cost effective; 9lf per capita GDP (7960 USD) < ICER <3 times of per capita GDP (23,880 USD), it is considered as cost effective; ${ }^{h}$ If ICER $>3$ times of per capita GDP (23,880 USD), it is considered not cost effective. Absolute dominated: An option is said to be dominated if it both costs more and is less effective than a comparator

ICER was less than per capita GDP at 7960 USD). The pathway of the most cost-effective strategy at different levels of willingness-to-pay thresholds as determined by the ICERs in different screening settings demonstrated that the dominant strategy gradually shifted from screening only to screening + HPV4, and finally screening + HPV9 (Fig. 2). When competing all strategies against each other, Screening 3, Screening $3+$ HPV4 and Screening $3+$ HPV9 were cost-efficient with ICERs below the threshold WTP. Screening $2+$ HPV9 and Screening $1+$ HPV9 were also on the cost-effective frontier but were not cost-effective as their ICERs (USD\$24,867/QALY; USD\$1,162,147/QALY) was over WTP (3 times of per capita USD\$23,880 USD) (Additional file 1: Figure S2).

Based on the natural history (no intervention), 1893 cases got cervical cancer and 348 cases died of it. HPV and genital wart infections occurred 895,739 times and 100,676 times, respectively. Among all 15 strategies, Screening $1+$ HPV9 showed the best preventative effect against HPV-related disease by reducing cervical cancer incidence by $34.39 \%$, cancer mortality cases by $35.95 \%$, HPV-Hr infection rate by $25.82 \%$, and genital wart incidence by $20.80 \%$ compared to no intervention. Among the three screening protocols, Screenings 1 and 2 showed no significant difference in their preventative effects against diseases related to HPV infection but were superior to Screening 3. Among the three vaccines, HPV9 showed the strongest preventative effect against HPVrelated disease compared to the other two in both the single and combination strategies (Table 2).

\section{Sensitivity analysis}

Variables with a range $\geq 1 \%$ of base case ICER (comparing each HPV9 combination strategy to HPV4 combination strategy) were listed in descending order (Fig. 3.1-3.3). The HPV4/9 combination strategies were both sensitive to efficacy of HPV4/9 for cervical cancer and genital warts, cost of HPV4/9, the discount rate of effectiveness, utility of warts, age of vaccination, screen coverage, and so on. Threshold analyses showed that when efficacy of HPV9 for genital warts/cervical cancer is smaller than $0.682 / 0.839$, cost of HPV9 more than \$567 USD, cost of HPV4 smaller than \$283 USD and efficacy of HPV4 for cervical cancer greater than 0.883 , ICER exceeds three times of GDP per capita (23,880 USD).

As HPV2 is the only accessible vaccine on mainland China so far, we also did a one-way sensitivity analysis for HPV2, comparing HPV2 combination strategies to 


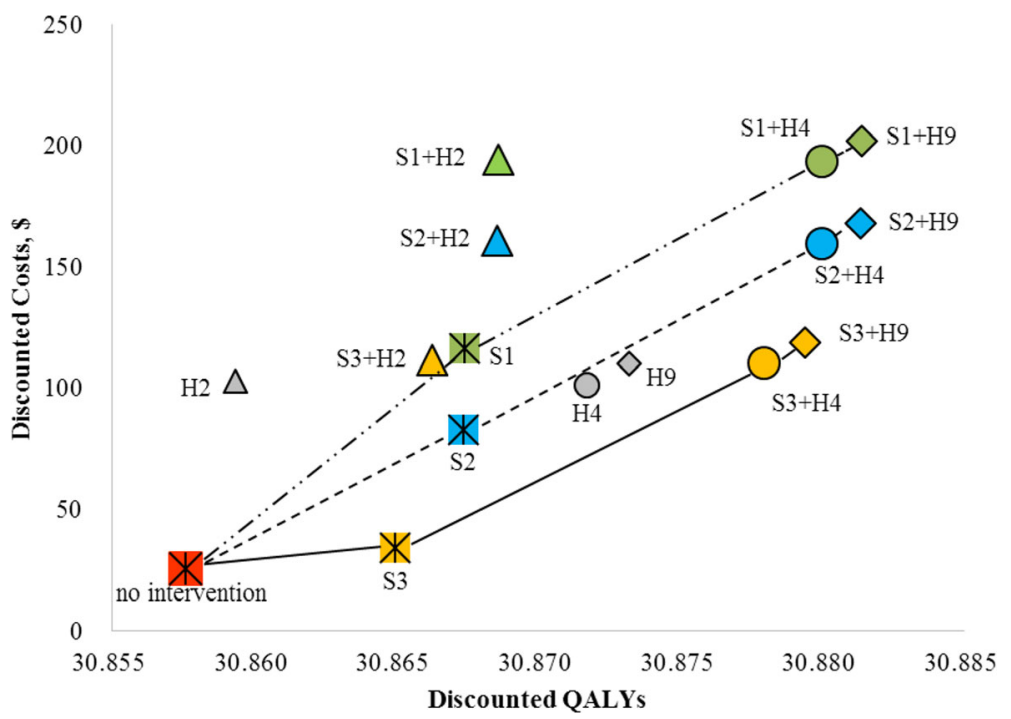

Fig. 2 Comparing Discounted cost and QALYs in different screening settings. Three lines stand for three different screening settings. S is short for screening; $\mathrm{H}$ is short for HPV vaccine. Green dot means strategies with screening1, blue dot means strategies with screening2 and yellow dot means strategies with screening3

screening alone (Additional file 1: Figure S3.1-S3.3). The results were mainly sensitive to the discount rate of effectiveness, screening coverage, discount rate of cost, utility of warts, CIN 3, age of vaccination, and so on. Since the screening coverage was important and public health meaningful among the results, we performed a threshold analysis of screening coverage from 10\% to $100 \%$, comparing it with willingness to pay (three times the GDP per capita of China). The combination of HPV2 with Screening 1 and Screening 2 showed similar values, and when screening coverage was increased to about $61 \%$, these strategies were found to be cost-effective. When screening was increased to $78 \%$, HPV2 combined with Screening 3 was also found to be considered acceptable (ICER doesn't exceed three times of GDP per capita).

\section{Discussion}

Past population-based screening programs demonstrated the impact of vaccination on reducing cervical cancer mortality and morbidity in both developed and developing countries $[45,46]$. Despite the overall decline in its disease burden, cervical cancer still remains the most lethal gynaecological cancer in China. Before HPV vaccines were approved for use on mainland China, the chief strategy for cervical cancer prevention and control was routine screening, targeting women at reproductive age for early detection and treatment. However, well-established evidence indicates that the success of such a program depends on adequate coverage of the population, which has often proved difficult to achieve [47]. As screening alone tends to be insufficient for combating the disease, and ideally primary prevention would be integrated with secondary measures, this study provided a perspective on the potential benefits of innovative vaccination strategies as an adjunct to the current screening program in China. The results of the first economic evaluation of an integrated strategy for the prevention and control of cervical cancer in China presented here will aid in informing policy makers at both the national and local levels.

The results of this study indicated that under baseline coverage of the vaccination and the screening programs, HPV2 vaccine, recently approved in China, is not cost effective either in the single vaccine protocol or in the screening combination strategies; however, the HPV4/9 strategy showed much better cost-effectiveness compared to HPV2 (with ICER of 5400 \$/QALY and 5434 \$/QALY respectively), and especially the strategy combining HPV9 with screening program showed best health impact on reducing the cervical cancer incidence and mortality rate, compared to that of the HPV2 vaccine combination strategy. These results were consistent with those of studies conducted in the United States [48, 49], which showed that the HPV9 vaccine resulted in a reduction in the incidence of genital warts among different populations $[26,50,51]$. After HPV 16/18, the most common HPV types among Chinese women with either a normal or abnormal cervical diagnosis were HPV 58,31 , and 52, which have been implicated in ICC among Chinese women and have accounted for $9.59 \% \sim 25.7 \%$ of ICC cases [30, 52]. They have now been added to the list of oncogenic types of HPV targeted by the secondgeneration HPV9 (HPV-31, 33, 45, 52 and 58). Furthermore, the potential benefits of HPV9 include a reduction in the incidence of vulvovaginal, penile, and anal tumours, 


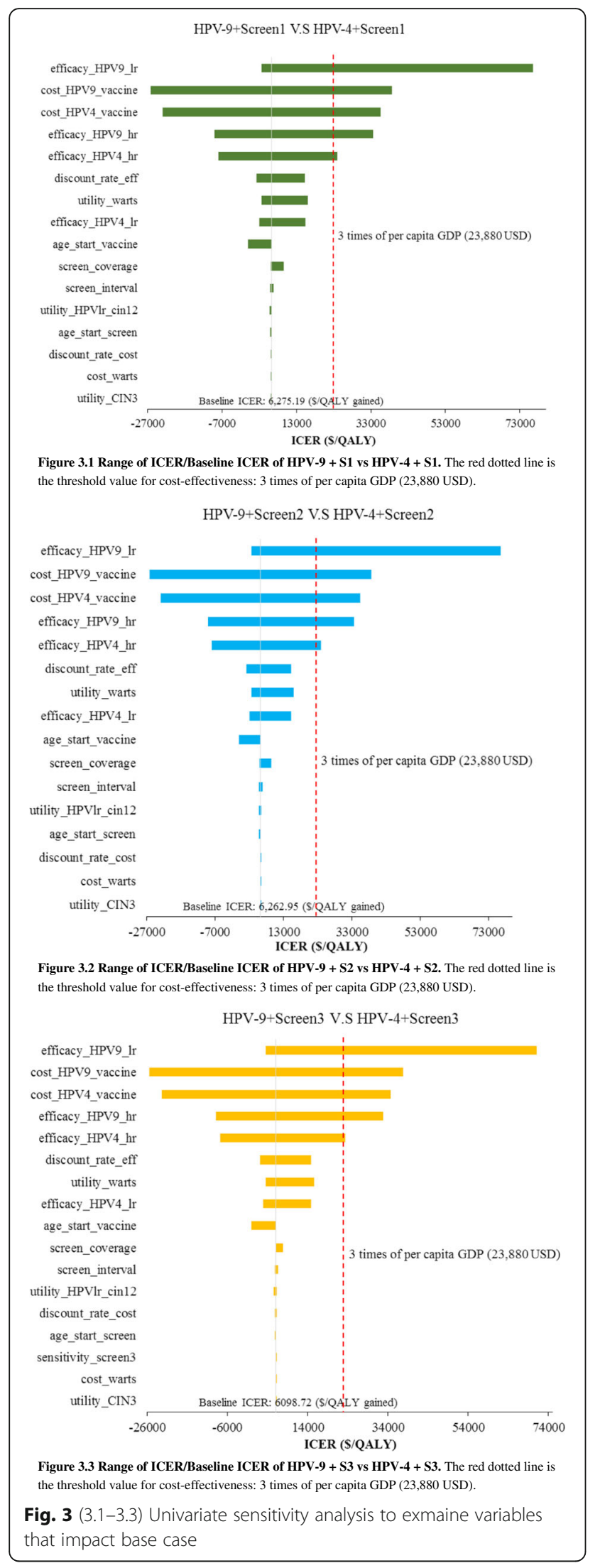

and paraneoplastic lesions arising in the lower-genital tract [53]. Among boys and male adolescents 55\% of anal and penile cancers are caused by the five oncogenic HPV types listed above. HPV9 vaccination not only covers these types specifically but also 68\% of all HPV types, and is thus expected to confer significant health benefits in males as well [54]. HPV9 will therefore be an invaluable adjunct to China's current vaccination program and should be considered seriously by public health policy makers.

Our results highlighted the validity of combining screening and vaccination. Combination strategies using HVP4/9 were cost-effective and able to confer greater health benefits than screening alone. Data from both developed and developing countries thus far have demonstrated that neither secondary prevention, such as screening alone, nor a conventional vaccination program can reduce the incidence of cervical cancers and warts or the disease burden [55]. Our results confirmed, in line with previous economic assessments, that integrated primary prevention (vaccination) and secondary prevention (screening program) were the most effective in terms of cost-effectiveness and health impact [55-57]. At the baseline level the HPV-9 + Screening 1 (Liquid-based cytology test + HPV DNA test) protocol was the most effective, having achieved a reduction of $34.39 \%$ in cancer incidence and of $35.95 \%$ in cancer deaths.

Vaccination is the primary preventative measure but effective only in those not yet infected by HPV [55]. Moreover, because immunity to HPV is primarily typespecific, the current generation of vaccines against a limited number of HPV types cannot provide complete protection [55]. Therefore, as a preliminary phase in the implementation of combination strategies, the current screening program should be strengthened by improving coverage for the general population [58]. Interestingly the recommended Screening 2 protocol (pap smear cytology test + HPV DNA test) showed almost the same effect as Screening 1 (liquid-based cytology test + HPV DNA test) in terms of reducing the incidence of cancer, HPV infections, and warts. The Screening 3 protocol (VIA), which cost much less and showed great costeffectiveness, is considered the best choice in medical resource-poor settings.

The different combination strategies examined in this study were flexible enough to be adapted to the wide geographical and socioeconomic diversity found in China according to the varying income levels and availability of medical resources. The Screening 3 or 2 protocols are recommended for low income settings, and if economic conditions permit, the screening plus vaccine strategy, which is likely to achieve have greater prevention impact, is also recommended even if HPV2 vaccine alone is available in mainland China so far.

The sensitivity analysis of HPV9 showed the robustness of its cost-effectiveness. The sensitivity analysis of 
HPV2, on the other hand, demonstrated that screening coverage crucially influenced the cost-effectiveness of different strategies. This study found that only when screening coverage increased to $60 \% \sim 70 \%$ did the HPV2 and screening combination strategies become economically feasible. In practice, coverage tends to be affected by various factors at both the social and individual level, such as affordability, the socioeconomic status of different regions, the educational level, and adherence of the target population to vaccine protocol recommendations [55]. Regarding the screening program, the current policy tends to be region driven and to focus on high risk populations, leading to a relatively low level of coverage in nonurban areas estimated to comprise approximately $20 \%$ of the population [59]. As for vaccination, most developing nations have experienced various challenges including coverage, accessibility, cold-chain transportation, and communication strategies to promote public acceptability [47]. Affordability is essential; high costs without public subsidy may restrict coverage especially among the poor and vulnerable. Educational interventions are also necessary to boost vaccination rates due to relatively poor HPV vaccine awareness among the Chinese [60]. Furthermore, because the vaccines are a relatively new development, the safety and the side effects remain a huge concern globally and affect the public's perceptions, and consequently the coverage, of any vaccination program [61, 62]. Especially for the newer HPV9 vaccine, more monitoring and surveillance are needed to establish safety and to maintain efficacy and cross-protection against oncogenic HPV types. These uncertainties should be carefully addressed in future studies for their potential impact on public health policy as well.

There were several limitations to this study. First, we did not model the transmission dynamics of HPV nor consider what protection might be conferred by HPV vaccinations against other HPV-related cancers like cervical adenocarcinoma, vulvar carcinoma, and laryngeal papillomatosis due to the lack of data. Consequently, the overall effectiveness of the vaccination program might have been underestimated, thus making the costeffectiveness of the HPV vaccine appear even more favourable if herd immunity or protection against other diseases existed $[63,64]$ especially in the case of HPV9, which confers wider coverage compared to the other two vaccines. Moreover, this study did not consider the cross-protective effects of vaccines, multiple HPV infections, and unidentified HPV types, which can influence the effectiveness of vaccines; instead we set a range for vaccine efficacy in our sensitivity analysis to determine the robustness of the model, showing that these variables did not significantly affect the ICERs under the current WTP threshold. In addition, women adhering to previous cervical screening tests may have shown better compliance with subsequent tests [13]. Nevertheless, in the absence of information on local contexts, we chose not to perform individually based modelling, nor did we consider the negative indirect effects.

\section{Conclusion}

We demonstrated that HPV4/9 vaccination for adolescent girls was highly cost-effective in China when integrated into the current screening strategies. Integrated vaccination and screening is recommended. HPV9 vaccination combined with Screening 1 (liquid-based cytology test + HPV DNA test) proved to be the best preventative strategy against cervical cancer and warts.

\section{Additional file}

Additional file 1: Table S1. Transfer probability used in the Markov model. Figure S1. Calibration result of age-specific incidence and mortality of cervical cancer. Figure S2. Comparing Discounted cost and QALYS with each strategy. $\mathrm{S}$ is short for screening; $\mathrm{H}$ is short for $\mathrm{HPV}$ vaccine. $\mathrm{Q}$ is short for QALY. Blue means strategies with screening1, yellow means strategies with screening2 and green screening3. Plots with grey outline means extended dominance. Figure S3.1. Spread of ICER/Baseline ICER of HPV-2 + S1 V.S S1. Figure S3.2. Spread of ICER/Baseline ICER of HPV-2 + S2 V.S S2. Figure S3.3. Spread of ICER/Baseline ICER of HPV-2 + S3 V.S S3. (DOC 288 kb)

\section{Abbreviations}

AIS: Adenocarcinoma In Situ; CIN: Cervical intraepithelial neoplasia; HPV: Human papillomavirus; VIA: Visual inspection with acetic acid; FIGO: International Federation of Gynaecology and Obstetrics; QALY: Quality-adjusted life year; CPI: Consumer price index; ICER: Incremental cost-effectiveness ratio; GDP: Gross domestic product; ICC: Invasive cervical cancer; WTP: Willingness to pay; HPV2, 4, 9: 2-, 4-, 9-valent HPV vaccine

\section{Acknowledgements}

The author is grateful to valuable comments on earlier versions of this paper; many thanks to the English editors in the Department of Health Policy, National Centre for Child Health and Development; and thanks to Prof Xu Lingzhong and Prof Li Shixue in Shandong University for their generous support when the author (XM) began this study during her master's program.

\section{Funding}

This research is supported by grants from the Japan Society for the Promotion of Sciences (16H06241), the National Centre for Child Health and Development (26-5), and the Chinese Natural Sciences Foundation (81202225).

Availability of data and materials

All the data can be found in the main paper or additional supporting files.

Authors' contributions

XM built the model, analysed data, and wrote the manuscript. RGT participated in the design of the study and wrote the manuscript. $\mathrm{XL}$ reviewed, reviewed and proofread the manuscript. $L W, B W, H L, C N, R M$, and TN participated in the discussion, review, and editing of the manuscript. All authors read and approved the final manuscript.

Ethics approval and consent to participate

As there was no direct patient participation in this study, ethic approval was exempted.

Consent for publication

All authors approved the final version of this article. 


\section{Competing interests}

All authors declare there were no potential competing interests relevant to this article.

\section{Publisher's Note}

Springer Nature remains neutral with regard to jurisdictional claims in published maps and institutional affiliations.

\begin{abstract}
Author details
${ }^{1}$ Department of Health Informatics, Kyoto University School of Public Health, Kyoto, Japan. ${ }^{2}$ Department of Health Policy, National Center for Child Health and Development, Okura 2-10-1, Setagaya-ku, Tokyo 157-8535, Japan. ${ }^{3}$ Department of Gynecology, Qilu Hospital of Shandong University, Jinan, China. ${ }^{4}$ College of Pharmacy, The University of Tennessee Health Science Center, Memphis, TN, USA. ${ }^{5}$ Medical Decision and Economic Group, Department of Pharmacy, Ren Ji Hospital affiliated with Medical School of Shanghai Jiao tong University, Shanghai, China. ${ }^{6}$ Nanjing Drum Tower Hospital affiliated Medical School of Nanjing University, Nanjing, China. ${ }^{7}$ Department of Education for Clinical Research, National Centre for Child Health and Development, Tokyo, Japan.
\end{abstract}

\section{Received: 20 December 2016 Accepted: 4 July 2017}

\section{Published online: 18 July 2017}

\section{References}

1. de Sanjose S, Diaz M, Castellsague X, Clifford G, Bruni L, Munoz N, et al. Worldwide prevalence and genotype distribution of cervical human papillomavirus DNA in women with normal cytology: a meta-analysis. Lancet Infect Dis. 2007;7:453-9.

2. Yang L, Parkin DM, Li LD, Chen YD, Bray F. Estimation and projection of the national profile of cancer mortality in China: 1991-2005. Br J Cancer. 2004;90:2157-66.

3. Shi JF, Canfell K, Lew JB, Qiao YL. The burden of cervical cancer in China: synthesis of the evidence. Int J Cancer. 2012;130:641-52.

4. Techakehakij W, Feldman RD. Cost-effectiveness of HPV vaccination compared with pap smear screening on a national scale: a literature review. Vaccine. 2008;26:6258-65.

5. Serrano B, Alemany L, Tous S, Bruni L, Clifford GM, Weiss T, et al. Potential impact of a nine-valent vaccine in human papillomavirus related cervical disease. Infect Agents Cancer. 2012;7:38

6. Chitnis D. Data on two-dose 9-valent HPV vaccinations encouraging, but more needed. https://www.pm360online.com/data-on-two-dose-9valenthpv-vaccinations-encouraging-but-more-needed/. Cited date: 24 Feb 2016.

7. Liu W. China approves GSK's HPV vaccine. http://www.chinadaily.com.cn/ china/2016-07/18/content 26131226.htm. Cited date: 18 July 2016.

8. GSK. GSK announces Cervarix ${ }^{\mathrm{TM}}$ approved in China to help protect women from cervical cancer. http://www.gsk-china.com/en-gb/media/pressreleases/2016/gsk-announces-cervarix-approved-in-china-to-help-protectwomen-from-cervical-cancer/. Cited date: 18 July 2016.

9. De Vuyst $\mathrm{H}$, Clifford GM, Nascimento MC, Madeleine MM, Franceschi S. Prevalence and type distribution of human papillomavirus in carcinoma and intraepithelial neoplasia of the vulva, vagina and anus: a meta-analysis. Int J Cancer. 2009;124:1626-36.

10. Merck \& CO. I. Highlights of prescribing information: GARDASIL ${ }^{\oplus}[$ Human papillomavirus quadrivalent (types $6,11,16$, and 18) vaccine, recombinant]. https://www.fda.gov/downloads/biologicsbloodvaccines/vaccines/ approvedproducts/ucm426457.pdf. Cited date: 28 Dec 2014.

11. Merck \& CO. I. Highlights of prescribing information: GARDASIL ${ }^{\oplus} 9$ (Human papillomavirus 9-valent vaccine, recombinant). www.merck.com/product/ patent/home.html. Cited date: 12 Mar 2015.

12. World Health Organization. Cervical cancer screening in developing countries: report of a WHO consultation. World Health Organization; 2002.

13. Dong Z. Chinese cancer screening and early detection and early treated guidelines (trail). Beijing: Peking University Medical Press; 2005.

14. Editorial. Women's health in rural China. Lancet. 2009:374:358.

15. Meng $R, M a W, X u Y$, Song $X, N i e S, X u H$, et al. Screening rates of cervical cancer, breast cancer and their influential factors in Guangdong Province. South China J Pre Med. 2010:8-10.

16. Li JM, Liu ZY, Ji HW, Fan YH, Wang W. Status of screening and KAP of cervical cancer in a community of Shenzhen. Chin J Health Educ. 2008;24:542-4.

17. Liu HR. Cervical cancer screening rates of its influential factors. World of mother and infant. 2015;
18. Liu Y-J, Zhang Q, Hu S-Y, Zhao F-H. Effect of vaccination age on costeffectiveness of human papillomavirus vaccination against cervical cancer in China. BMC Cancer. 2016;16:1-11.

19. Zhang Q, Liu Y-J, Hu S-Y, Zhao F-H. Estimating long-term clinical effectiveness and cost-effectiveness of HPV 16/18 vaccine in China. BMC Cancer. 2016;16:848.

20. Levin CE, Sharma M, Olson Z, Verguet S, Shi J-F, Wang S-M, et al. An extended cost-effectiveness analysis of publicly financed HPV vaccination to prevent cervical cancer in China. Vaccine. 2015;33:2830-41.

21. Liu PH, Hu FC, Lee PI, Chow SN, Huang CW, Wang JD. Cost-effectiveness of human papillomavirus vaccination for prevention of cervical cancer in Taiwan. BMC Health Serv Res. 2010;10:11.

22. Elbasha EH, Dasbach EJ, Insinga RP. Model for assessing human papillomavirus vaccination strategies. Emerg Infect Dis. 2007;13:28-41.

23. Myers ER, McCrory DC, Nanda K, Bastian L, Matchar DB. Mathematical model for the natural history of human papillomavirus infection and cervical carcinogenesis. Am J Epidemiol. 2000;151:1158-71

24. Longde W, Yu P. Technical proposal for screening and early detection and treatment of cancer in China. People's medical publishing house; 2009.

25. Shi JF, Chen JF, Canfell K, Feng XX, Ma JF, Zhang YZ, et al. Estimation of the costs of cervical cancer screening, diagnosis and treatment in rural Shanxi Province, China: a micro-costing study. BMC Health Serv Res. 2012:12:123.

26. Shi JF, Xiang W, Qiao YL. A review of health economic evaluation on cervical cancer screening by visual inspection with acetic acid. Zhonghua Liu Xing Bing Xue Za Zhi. 2009;30:87-90.

27. Kulasingam SL, Myers ER. Potential health and economic impact of adding a human papillomavirus vaccine to screening programs. JAMA. 2003;290(6):781-9.

28. Jufang S. Cost-effectiveness on various modalities of cervical cancer screening in rural China: Chinese academy of medical sciences \& Peking union medical college; 2009 .

29. GlaxoSmithKline. Highlights of prescribing information: CERVARIX [Human papillomavirus bivalent (types 16 and 18) vaccine, recombinant]. http://us. gsk.com/products/assets/us_cervarix.pdf. Cited date: 28 Dec 2014.

30. Wen C. An epidemic study on HPV distribution in genital warts, invasive cervical cancer in China: Chinese academy of medical sciences \& Peking union medical college; 2010.

31. Olsson SE, Villa LL, Costa RL, Petta CA, Andrade RP, Malm C, et al. Induction of immune memory following administration of a prophylactic quadrivalent human papillomavirus (HPV) types 6/11/16/18 L1 virus-like particle (VLP) vaccine. Vaccine. 2007;25:4931-9.

32. Zhao FH, Lin MJ, Feng C, Hu SY, Rong Z, Belinson JL, et al. Performance of high-risk human papillomavirus DNA testing as a primary screen for cervical cancer: a pooled analysis of individual patient data from 17 populationbased studies from China. Lancet Oncol. 2010;11:1160-71.

33. Dongyan L. Health economic evaluation and economic feasibility research of cervical cancer screening in rural areas: medical school of Dalian; 2010.

34. Zhao ZM, Pan XF, Lv SH, Xie Y, Zhang SK, Qiao YL, et al. Quality of life in women with cervical precursor lesions and cancer: a prospective, 6-month, hospital-based study in China. Chin J Cancer. 2014;33:339-45.

35. Molassiotis A, Chan CW, Yam BM, Chan SJ. Quality of life in Chinese women with gynaecological cancers. Support Care Cancer. 2000;8:414-22.

36. Shi JF, Kang DJ, Qi SZ, Wu HY, Liu YC, Sun LJ, et al. Impact of genital warts on health related quality of life in men and women in mainland China: a multicenter hospital-based cross-sectional study. BMC Public Health. 2012;12:153.

37. Qi SZ, Wang SM, Shi JF, Wang QQ, Chen XS, Sun LJ, et al. Human papillomavirus-related psychosocial impact of patients with genital warts in China: a hospital-based cross-sectional study. BMC Public Health. 2014;14:1-8.

38. People Daily. What is HPV? http://weibo.com/rmrb?is_hot=1. Cited date: 11 Mar 2016

39. Medscape. HPV 9: The Latest ACIP Guidance. http://www.medscape.com/ viewarticle/841676. Cited date: 23 Mar 2015

40. Chinses anti-cancer association. Early screen of cervical cancer [in Chinese] http://www.caca.org.cn/system/2008/07/31/010009930.shtml. Cited date: 15 Dec 2008.

41. National Comprehensive Cancer Network. NCCN Clinical Practice Guidelines in Oncology http://www.nccn.org/professionals/physician_gls/f_guidelines.asp. 2013

42. World Health Organization. Macroeconomics and Health: Investing in Health for Economic Development. Report of the Commission on Macroeconomics and Health. Geneva: WHO; 2001.

43. National Bureau of Statistics of China Gross Domestic Product http//data. stats.gov.cn/ks.htm?cn=C01\&zb=A0501. Cited date: 1 Dec 2015. 
44. National Office for Cancer Prevention and Control NCfCR. Disease Prevnetion and Control Bureau, MOH. Chinese Cancer Registry Annual Report (2011). Beijing: Military Medical Science Press; 2011.

45. Mandelblatt JS, Lawrence WF, Womack SM, Jacobson D, Yi B, Hwang YT, et al. Benefits and costs of using HPV testing to screen for cervical cancer. JAMA. 2002;287:2372-81.

46. Chen MK, Hung HF, Duffy S, Yen AM, Chen HH. Cost-effectiveness analysis for pap smear screening and human papillomavirus DNA testing and vaccination. J Eval Clin Pract. 2011;17:1050-8.

47. Kane MA, Serrano B, de Sanjose S, Wittet S. Implementation of human papillomavirus immunization in the developing world. Vaccine. 2012; 30(Suppl 5):F192-200.

48. Chesson HW, Markowitz LE, Hariri S, Ekwueme DU, Saraiya M. The impact and cost-effectiveness of nonavalent HPV vaccination in the United States: estimates from a simplified transmission model. Human vaccines \& immunotherapeutics. 2016:1-10.

49. Brisson M, Laprise JF, Chesson HW, Drolet M, Malagon T, Boily MC, et al. Health and economic impact of switching from a 4-Valent to a 9-Valent HPV vaccination program in the United States. J Natl Cancer Inst. 2016;108

50. Sorapop K, Nicole Gastineau C, Kim JJ. Potential benefits of secondgeneration human papillomavirus vaccines. PLoS One. 2012;7:e48426.

51. Lee VJ, Tay SK, Teoh YL, Tok MY. Cost-effectiveness of different human papillomavirus vaccines in Singapore. BMC Public Health. 2011;11:203.

52. Bao YP, Li N, Smith JS, Qiao YL. Human papillomavirus type-distribution in the cervix of Chinese women: a meta-analysis. Int J STD AIDS. 2008;19:106-11.

53. Mariani L, Bonanni $\mathrm{P}$, Castiglia $\mathrm{P}$, Chiamenti G, Conforti G, Conversano M, et al. Prevention of HPV cancer related through HPV-9: state of the art, potential benefits and open issues. Igiene e sanita pubblica. 2015;71:631-52

54. Wieland U, Brockmeyer NH, Kreuter A. A 9-Valent HPV Vaccine in Women. N Engl J Med. 2015;372:2566.

55. Liu X, Feng A, Cui Y, Tobe RG. Prevention of human papillomavirus (HPV) infection and cervical cancer in China: how does HPV vaccination bring about benefits to Chinese women? Biosci Trends. 2013:7:159-67.

56. Diaz M, Kim JJ, Albero G, Sanjosé SD, Clifford G, Bosch FX, et al. Health and economic impact of HPV 16 and 18 vaccination and cervical cancer screening in India. Br J Cancer. 2008;99:230-8.

57. Canfell K, Shi JF, Lew JB, Walker R, Zhao FH, Simonella L, et al. Prevention of cervical cancer in rural China: evaluation of HPV vaccination and primary HPV screening strategies. Vaccine. 2011;29:2487-94.

58. Zhai L, Tumban E. Gardasil-9: a global survey of projected efficacy. Antivir Res. 2016.

59. ChinalRN. New opportunities of cervical cancer prevention and control to China in 2014 http://www.chinairn.com/news/20140506/124657368.shtml. Cited date: 30 Dec 2014.

60. Zhang Y, Wang Y, Liu L, Fan Y, Liu Z, Wang Y, et al. Awareness and knowledge about human papillomavirus vaccination and its acceptance in China: a meta-analysis of 58 observational studies. BMC Public Health. 2016;16:216.

61. Stokley S, Jeyarajah J, Yankey D, Cano M, Gee J, Roark J, et al. Human papillomavirus vaccination coverage among adolescents, 2007-2013, and postlicensure vaccine safety monitoring, 2006-2014-United States. MMWR Morb Mortal Wkly Rep. 2014;63:620-4.

62. Handler NS, Handler MZ, Majewski S, Schwartz RA. Human papillomavirus vaccine trials and tribulations: vaccine efficacy. J Am Acad Dermatol. 2015; 73:759-67. quiz 67-8

63. Chesson HW, Ekwueme DU, Saraiya M, Markowitz LE. Cost-effectiveness of human papillomavirus vaccination in the United States. Emerg Infect Dis. 2008;14:244.

64. Brisson M, Edmunds WJ. Economic evaluation of vaccination programs: the impact of herd-immunity. Med Decis Mak. 2003:23:76-82.

\section{Submit your next manuscript to BioMed Central and we will help you at every step:}

- We accept pre-submission inquiries

- Our selector tool helps you to find the most relevant journal

- We provide round the clock customer support

- Convenient online submission

- Thorough peer review

- Inclusion in PubMed and all major indexing services

- Maximum visibility for your research

Submit your manuscript at www.biomedcentral.com/submit
Biomed Central 